Proceedings of the WELCOME Scientific Meeting on Hybrid Nanostructures, Toruń, Poland, August 28-31, 2011

\title{
Controlling Structural and Functional Features of Photosynthetic Antenna
}

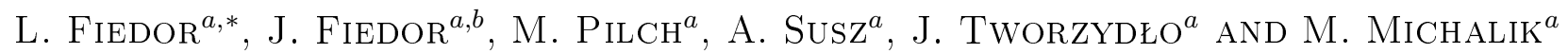 \\ ${ }^{a}$ Faculty of Biochemistry, Biophysics and Biotechnology, Jagiellonian University \\ Gronostajowa 7, 30-387 Kraków, Poland \\ ${ }^{b}$ AGH University of Science and Technology, al. A. Mickiewicza 30, 30-059 Kraków, Poland
}

In order to gain control over the assembly and functioning of photosynthetic antenna, we have developed methods to manipulate pigment composition of bacterial LH1 complexes via their reconstitution with modified bacteriochlorophylls or carotenoids, major photoactive cofactors of these antennae. In the present work we show how the reconstitution and pigment exchange approach is applied to control structural and functional parameters of LH1 and its subunits. The size of the subunits and the energy of the first excited singlet state can be controlled via the use of detergent while the thermodynamics of LH1 formation can be modified using carotenoids and/or a co-solvent. Carotenoids affect the efficiency of the intracomplex energy transfer, while the replacement of native bacteriochlorophyll $a$ with its Ni-substituted analog allows one to control the excited state properties of LH1. These results show that LH1 is a very promising model system applicable for the design of bio-inspired device performing solar energy conversion.

PACS: 87.15.nr, 87.15.Cc, 87.64.ks

\section{Introduction}

The photosynthetic processes in purple photosynthetic bacteria take place in a specialized photochemical nanodevice, a complex composed of reaction center (RC) and light harvesting antenna 1 (LH1), which carries out light harvesting and charge separation across the photosynthetic membrane. The in vitro reconstitution studies show that both the LH1 and LH1-RC complexes are self-organizing systems but the knowledge of the factors and forces that govern these systems is still limited [1-5]. Bacteriochlorophylls (BChls) and carotenoids (Crts, examples shown in Fig. 1) are required for the cor-

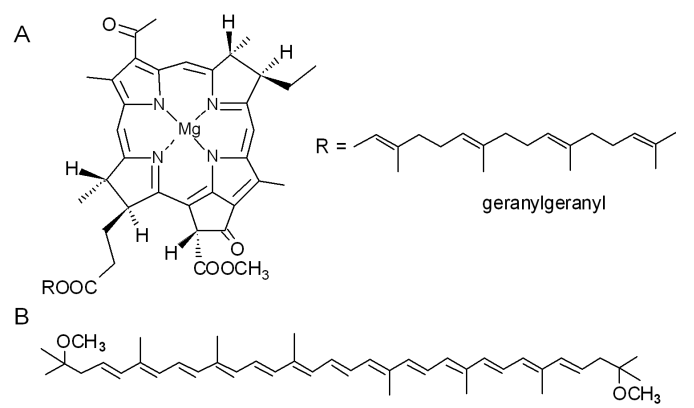

Fig. 1. The formulae of (A) bacteriochlorophyll $a$ and (B) spirilloxanthin, the main photosynthetic pigments in Rhodospirillum rubrum.

rect assembly of bacterial photosystems [6], where they function as photoactive as well as structural cofactors. Because bacteriochlorophylls have excellent photosensitizing properties and act as efficient singlet oxygen and

* corresponding author; e-mail: leszek.fiedor@uj.edu.pl other reactive oxygen species generators $[7,8]$, Crts in all photosystems also play a very important role as intrinsic photoprotectors [9].

LH1 is composed of 15-16 heterodimeric subunits of short hydrophobic polypeptides termed $\alpha$ and $\beta$, hosting a circular array of 30-32 strongly interacting BChls $[1,10,11]$. The recently resolved crystal structure of the LH1-RC complex reveals that the circular symmetry of the complex is broken by an additional transmembrane helix, creating a gap in the LH1 cylinder near the ubiquinone binding site of the RC [12]. BChls are in van der Waals contact with Crts, mainly via the long alcohol residues esterifying the C-17 propionic acid side group [13]. The occurrence of strong pigment-pigment interactions in the densely packed rings of BChls is obvious [14], but the degree of exciton delocalization and its dynamics pose questions. The extremes spread from complete delocalization $(n=16-32)$, to smaller basic units $(n=2)$, which are similar to the special pair of BChls in RC [14-16].

The LH1 antenna, being an assembly of relatively simple identical subunits, is perhaps the simplest type of photosynthetic antenna complex and the reversibility of its association-dissociation in micellar media renders it a very attractive model complex $[2,17,18]$. In the present paper, the techniques which enable for construction of model LH1 complexes from chemically modified components are described and discussed.

\section{Results and discussion \\ 2.1. Assembly of LH1}

LH1 can be dissociated into the subunits of well defined sizes [19-22] and spectral properties, in particular, the position of the lowest energy transition $Q_{Y}$, as indicated in Fig. 2. The subunits in LH1 are held together by weak interactions and their reversible dissociation can 
be induced by detergents. The $\mathrm{LH} 1 /$ micelle ratio determines the equilibrium between the subunits and assembled antenna according to the equation

$$
\mathrm{B} 780_{d} \leftrightarrow \mathrm{B} 820_{d} \leftrightarrow \mathrm{B} 870_{d},
$$

where, in the absence of Crts, B780, B820, and B870 are the monomeric and dimeric subunits, and a fully assembled antenna, respectively. The symbol $d$ denotes the involvement of the detergent. As discussed below, at a fixed detergent concentration, the same effect can be achieved by varying the temperature $[17,23]$.
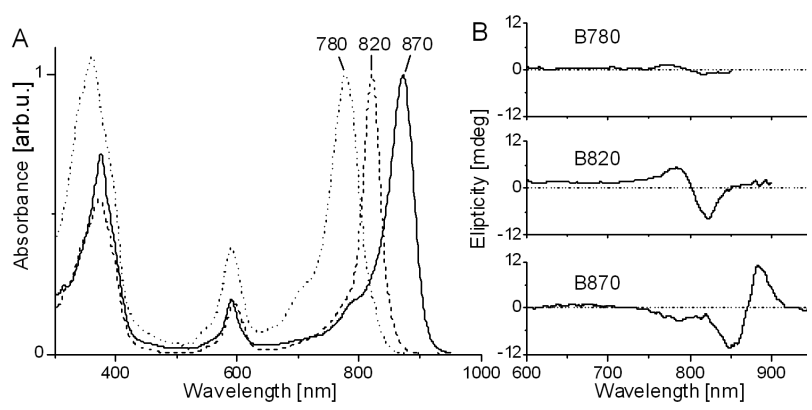

Fig. 2. The electronic absorption (A) and circular dichroism (B) spectra of the carotenoid-less B780, B820, and B870 forms of the LH1 antenna from Rhodospirillum rubrum.

In the monomeric subunits, B780, specific His residues of the $\alpha$ and $\beta$ polypeptides coordinatively interact with the central $\mathrm{Mg}$ ions of BChla. In addition, BChla is involved in other interactions with the apoprotein, as indicated by the shift of the $Q_{Y}$ band from 770 to $780 \mathrm{~nm}$. The monomeric subunits assembly to the dimeric (or tetrameric) B820 [20, 24], in part due to pigment-pigment interactions $(\pi-\pi$ stacking) and to pigment-polypeptide and polypeptide-polypeptide hydrophobic interactions, probably involving the phytyl moiety $[13,25]$. The characteristic red shift of the $Q_{Y}$ band originates from exciton coupling in the $\alpha \beta$ (BChla) $)_{2}$-heterodimers. B780 and B820 aggregate further to larger oligomers and the formation of B870 ring terminates the folding of LH1.

The shift of the $Q_{Y}$ transition to $870 \mathrm{~nm}$ is caused by excitonic interactions between strongly coupled BChla molecules within the B870 array, which is reflected in the characteristic shape of the circular dichroism spectra (Fig. 2). The ring of BChla molecules in LH1 from Rhodospirillum rubrum may constitute up to 32 pigment molecules but smaller assemblies with similar spectral properties can be formed as well [22]. The B780, B820, and B870 forms interact with Crts [23, 26, 27], which increases the driving force for the assembly (see below). These interactions must be mainly of hydrophobic character because carotenes, such as neurosporene and lycopene, also induce the oligomerization [23, 28]. Crts seem to enhance excitonic coupling between BChla molecules in LH1, as indicated in the shift of $Q_{Y}$ to $880 \mathrm{~nm}$ induced by the binding of Crts $[6,27]$.

\subsection{Driving force for LH1 assembly}

The values of basic thermodynamic parameters of LH1 formation from the subunits (Eq. (1)) were determined using the van 't Hoff method, based on the deconvolution of the absorption spectra of reconstitution mixture, recorded at various temperatures [23]. In the absence of Crt and co-solvent, the $\Delta H^{\circ}$ of LH1 formation in $0.03 \%$ LDAO reaches almost $-700 \mathrm{~kJ} / \mathrm{mol}$ and it increases to $-1160 \mathrm{~kJ} / \mathrm{mol}$ upon the addition of $15 \%$ acetone, and further to $-1900 \mathrm{~kJ} / \mathrm{mol}$ in the presence of spirilloxanthin [23]. In each case, the $\Delta G^{\circ}$ of the complex assembly amounts to $-1050 \mathrm{~kJ} / \mathrm{mol}$, which indicates that the increase in the energetic effect is compensated by the drop in $\Delta S^{\circ}$.

Crts are involved in strong interactions with the LH1 subunits from the very moment of being introduced into the reconstitution system, as reflected in the thermodynamic parameters of LH1 formation [23]. The addition of Crt causes a rise in the inter-subunit interaction energy but it is compensated by entropy loss, which originates from an ordering effect due to a larger interaction energy. The incorporation of Crt into the complex also reduces the disorder in the system. The effect of co-solvent seem to be related to the permittivity of the medium; it is initially very high $(\approx 80)$ and it lowers in the presence of acetone. Apparently, this enhances electrostatic interactions between LH1 subunits $\left(=\right.$ larger $\Delta H^{\circ}$ ), reflected in a $25 \%$ higher efficiency of the intracomplex Crt $\rightarrow$ BChl energy transfer [23].

\subsection{Intracomplex energy transfer}

The efficiency of the intracomplex energy transfer in LH1 can be controlled by structure of the bound Crt. A series of LH1 complexes incorporating various Crts (neurosporene, spheroidene, lycopene, anhydrorhodovibrin and spirilloxanthin) was obtained, using the Crt exchange approach $[28,29]$. The correctness of LH1 assembly is confirmed by the high efficiency of the intracomplex Crt $\rightarrow$ BChl singlet energy transfer (Fig. 3). A comparison of the fluorescence excitation spectra showed that the efficiency of Crt $\rightarrow$ BChla energy transfer decreases gradually from 90 to $35 \%$ along the series, very close to the values obtained by ultrafast absorption spectroscopy $[28,29]$. As the number of conjugated double bonds in Crt increases above 11, not all low-lying singlet states are available as channels for energy transfer to BChl.

\subsection{Excited state properties}

The properties of the excited state of LH1 can be modulated using the Ni-substituted BChla as the ultrafast excitation trap [30, 31]. The incorporation of Ni-BChla into LH1 causes only minor changes in the ground state features of the modified complex but has dramatic effects on its emission properties, as shown in Fig. 4. The emission intensity sharply decreases in the presence of even small amounts of Ni-BChla in LH1. In LH1 from Rhodobacter sphaeroides, already the incorporation of $3.2 \%$ Ni-BChla 


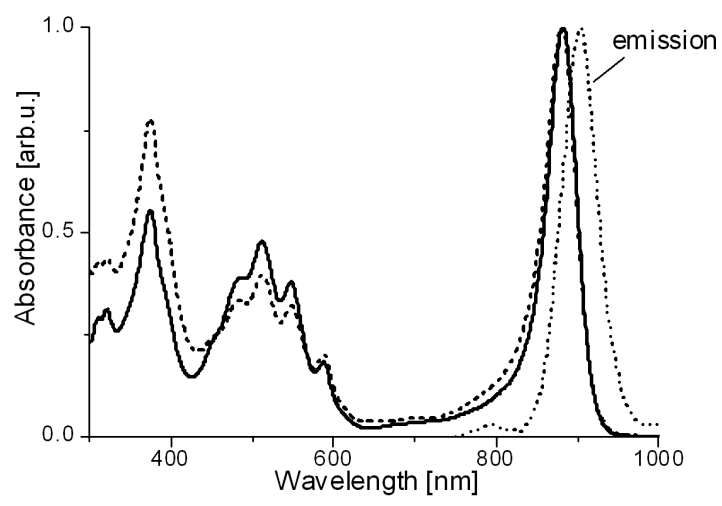

Fig. 3. The absorption spectra of the native (solid line) and reconstituted (dashed line) LH1 antenna from Rhodospirillum rubrum, overlapped with their fluorescence emission spectrum (dotted line), obtained using the excitation at $500 \mathrm{~nm}$.

causes a nearly $50 \%$ quenching and at $20 \%$ Ni-BChla the emission drops to zero [31]. The emission decays monoexponentially with practically the same time constant of $850 \pm 100$ ps [31]. However, the emission signals decrease strongly upon incorporation of Ni-BChla, in excellent agreement with the fluorescence quenching. A similarly strong quenching effect was observed in LH1 from Rhodospirillum rubrum, reconstituted from Ni-BChla containing B20 subunits (B820 $\mathrm{Ni}$, Fig. 4).

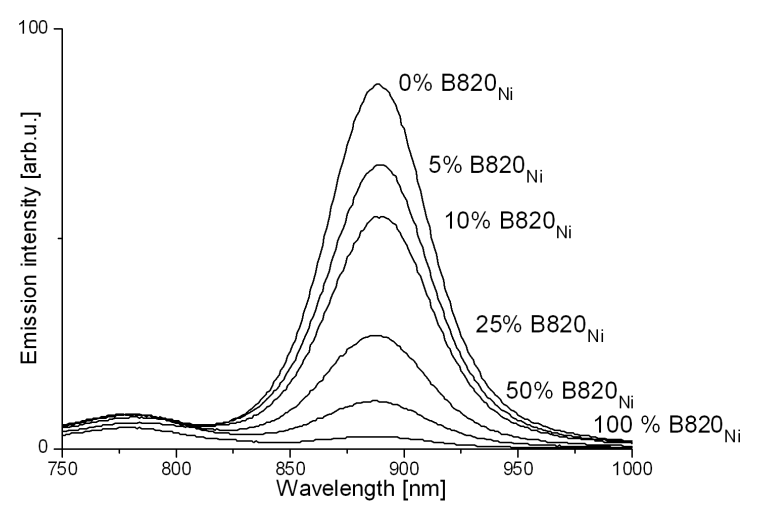

Fig. 4. The emission profiles, normalized to the same absorbance at the excitation wavelength $(840 \mathrm{~nm})$, of the B870 complexes obtained from Ni-BChla substituted B820 mixed at varying ratios with unmodified B820.

The major conclusions from femtosecond pump-probe absorption spectroscopy measurements were that the trapping occurs on $\approx 60$ fs time scale [30]. One Ni-BChla per 20 BChls is sufficient for complete excitation deactivation via the $60 \mathrm{fs}$ channel. It excludes any localized excitation, monomeric as well as dimeric, and subsequent Förster hopping in LH1. These findings suggest that the excitation is delocalized over at least $10 \mathrm{BChl}$ molecules or even the entire LH1 unit.

\section{Materials and methods}

\subsection{Purification of native LH1 antenna}

LH1 was prepared from Rhodospirillum rubrum (S1 strain) as described previously [28]. The bacterial chromatophores were treated with $0.45 \% \operatorname{LDAO}(\mathrm{v} / \mathrm{v})$ and ultracentrifuged to separate LH1 from RC. For the final purification, the pellet containing LH1 was solubilized in $2.35 \% \beta$-OG $(\mathrm{w} / \mathrm{v})$ and LH1 was isolated by column chromatography on DEAE-cellulose (DE52 Whatman), equilibrated in $0.8 \% \beta-\mathrm{OG}$.

\subsection{Reconstitution of LH1 with Crt}

The insertion of spirilloxanthin into LH1 was carried out according to a previously published method [28]. The Crt-depleted antenna was obtained from freeze-dried and benzene-extracted chromatophores from Rhodospirillum rubrum by solubilization in $0.3 \% \operatorname{LDAO}(\mathrm{v} / \mathrm{v})$. The B780-B820-B870 mixture was titrated with acetone solution of the pigment. The volume of the reconstitution mixture was kept constant and the progress of reconstitution was monitored by absorption and emission spectroscopy. After the complex stabilization $\left(12 \mathrm{~h}\right.$ at $4^{\circ} \mathrm{C}$ ), the spirilloxanthin-LH1 complex was purified by column chromatography on a DEAE-cellulose in the presence of $0.025 \%$ LDAO.

\subsection{Isolation of the B820 subcomplex}

The Crt-depleted chromatophores were treated with $20 \%$ aqueous $\beta$-OG (v/w) until solubilization. After centrifugation, the B820 subunits were purified on a DEAE-sepharose (Fast Flow, Pharmacia), pre-equilibrated in $1 \% \beta$-OG $(10 \mathrm{mM} \mathrm{NaCl} / 20 \mathrm{mM}$ TRIS. $\mathrm{HCl}$ buffer, $\mathrm{pH}$ 7.8), as described previously [27].

To achieve a partial substitution of native BChla in B820 with Ni-BChla, the solubilized antenna subunits were incubated with a portion of the $\mathrm{Ni}$ derivative added as acetone solution. The $\mathrm{B} 820_{\mathrm{Ni}}$ subunits were purified in the same way as the unsubstituted B820. To obtain the Ni-BChla-substituted B870 complexes, the unsubstituted $\mathrm{B} 820$ were mixed with $\mathrm{B} 820_{\mathrm{Ni}}$ at varying ratios, ranging from $0 \%$ to $29 \%$ of $\mathrm{B} 820_{\mathrm{Ni}}$, and their oligomerization was induced by lowering the detergent concentration below the CMC (0.4\%) and storing overnight at $4{ }^{\circ} \mathrm{C}$.

\subsection{Pigment isolation and modification}

BChla was isolated from the carotenoidless strain R26.1 of Rhodobacter sphaeroides according to a method of Omata et al. [32], with some modifications [33]. The cells were extracted with methanol, the pigments transferred to acetone and separated on a DEAE-sepharose column, pre-equilibrated in acetone. The fraction of pure BChla was eluted with $20 \%$ methanol in acetone. The central $\mathrm{Mg}^{2+}$ ion of BChla was replaced by $\mathrm{Ni}^{2+}$ using the transmetalation method via the Cd-BChla intermediate according to Hartwich et al. [34]. The purity of the final product was confirmed by TLC and HPLC. 
Spirilloxanthin was extracted from the cells of the Rhodospirillum rubrum and purified by low-pressure column chromatography on $\mathrm{Ca}(\mathrm{OH})_{2}$, using acetone/benzene mixture for elution, and repurified by recrystallization in $n$-hexane [35]. All preparative steps were carried out in dim light.

\subsection{Electronic absorption, emission and CD spectra}

The electronic absorption spectra were measured in $1 \mathrm{~mm}$ quartz cells using a Cary 50 (Varian) spectrophotometer. Circular dichroism spectra were recorded in the near IR region at room temperature in $1 \mathrm{~cm}$ quartz cells with a J-815 spectropolarimeter (JASCO). The steady state fluorescence emission measurements were performed using a FluoroMax-P spectrofluorometer (Jobin-Yvon, Horiba) equipped with a sample temperature controller. The excitation wavelength was set to $840 \mathrm{~nm}$ and fluorescence spectra were recorded in the range between 850 and $1000 \mathrm{~nm}$, averaging three consecutive measurements. All samples were diluted to the same absorbance $(0.25)$ at $840 \mathrm{~nm}$ and the emission spectra were measured in a $2 \mathrm{~mm}$ thick quartz cell.

\section{Acknowledgments}

The work was supported by a research grant from the Foundation for Polish Science (TEAM/2010-5/3). The Faculty of Biochemistry, Biophysics and Biotechnology of the Jagiellonian University is a beneficiary of the structural funds from the European Union (grant No: POIG.02.01.00-12-064/08 — "Molecular biotechnology for health").

\section{References}

[1] C.M. Davis, P.S. Parkes-Loach, C.K. Cook, K.A. Meadows, M. Bandilla, H. Scheer, P.A. Loach, Biochemistry 35, 3072 (1996).

[2] C.M. Davis, P.L. Bustamante, J.B. Todd, P.S. Parkes-Loach, P. McGlynn, J.D. Olsen, L. McMaster, C.N. Hunter, P.A. Loach, Biochemistry 36, 3671 (1997).

[3] R.J. Pugh, P. McGlynn, M.R. Jones, C.N. Hunter, Biochim. Biophys. Acta 1366, 301 (1998).

[4] C. Jungas, J.-L. Ranck, J.-L. Rigaud, P. Joliot, A. Vermeglio, EMBO J. 18, 534 (1999).

[5] R.N. Frese, J.D. Olsen, R. Branvall, W.H.J. Westerhuis, C.N. Hunter, R. van Grondelle, Proc. Natl. Acad. Sci. USA 97, 5197 (2000).

[6] C.M. Davis, P.L. Bustamante, P.A. Loach, J. Biol. Chem. 270, 5793 (1995).

[7] L. Fiedor, A.A. Gorman, I. Hamblett, V. Rosenbach-Belkin, Y. Salomon, A. Scherz, I. Tregub, Photochem. Photobiol. 58, 506 (1993).

[8] J. Fiedor, L. Fiedor, J. Winkler, A. Scherz, H. Scheer, Photochem. Photobiol. 74, 64 (2001).

[9] Carotenoids in Photosynthesis, Eds. A. Young, G. Britton, Chapmann \& Hall, London 1993.

[10] S. Karrasch, P.A. Bullough, R. Ghosh, EMBO J. 14, 631 (1995).
[11] C. Francke, J. Amesz, Photosynth. Res. 46, 347 (1995).

[12] A.W. Roszak, T.D. Howard, J. Southall, A.T. Gardiner, C.J. Law, N.W. Isaacs, R.J. Cogdell, Science 302, 1969 (2003).

[13] P.S. Parkes-Loach, A.P. Majeed, C.J. Law, P.A. Loach, Biochemistry 43, 7003 (2004).

[14] A. Scherz, V. Rosenbach-Belkin, J. Fisher, Proc. Natl. Acad. Sci. USA 87, 5430 (1990).

[15] J.A. Leegwater, J. Phys. Chem. 100, 14403 (1996).

[16] M. Chachisvilis, O. Kühn, T. Pullerits, V. Sundström, J. Phys. Chem. 101, 7275 (1997).

[17] J.N. Sturgis, B. Robert, J. Mol. Biol. 238, 445 (1994).

[18] P. Braun, L. Fiedor, in The Purple Phototrophic Bacteria, Eds. C.N. Hunter, F. Daldal, M.C. Thurnauer, J.T. Beatty, Springer, Dordrecht 2009, p. 913.

[19] R. Ghosh, H. Hauser, R. Bachofen, Biochemistry 27, 1004 (1988).

[20] M.C. Chang, P.M. Callahan, P.S. Parkes-Loach, T.M. Cotton, P.A. Loach, Biochemistry 29, 421 (1990).

[21] V. Arluison, J. Seguin, B. Robert, Biochemistry 41, 11812 (2002).

[22] A. Pandit, I.H.M. van Stokkum, S. Georgakopoulu, G. van der Zwan, R. van Grondelle, Photosynth. Res. 75, 235 (2003).

[23] J. Fiedor, M. Pilch, L. Fiedor, J. Phys. Chem. B 113 , 12831 (2009).

[24] A.P. Vegh, B. Robert, FEBS Lett. 528, 222 (2002).

[25] Z.-Y. Wang, Y. Muraoka, M. Shimonaga, M. Kobayashi, T. Nozawa, J. Am. Chem. Soc. 124, 1072 (2002).

[26] A. Moskalenko, O. Toropygina, N. Kuznetsova, Z. Naturforsch. 51, 309 (1996).

[27] L. Fiedor, H. Scheer, J. Biol. Chem. 280, 20921 (2005).

[28] L. Fiedor, J. Akahane, Y. Koyama, Biochemistry 43 , 16487 (2004).

[29] J. Akahane, F.S. Rondonuwu, L. Fiedor, Y. Watanabe, Y. Koyama, Chem. Phys. Lett. 393, 184 (2004).

[30] L. Fiedor, H. Scheer, C.N. Hunter, F. Tschirschwitz, B. Voigt, J. Ehlert, E. Nibbering, D. Leupold, T. Elsaesser, Chem. Phys. Lett. 319, 145 (2000).

[31] L. Fiedor, D. Leupold, K. Teuchner, B. Voigt, C.N. Hunter, A. Scherz, H. Scheer, Biochemistry 40, 3737 (2001).

[32] T. Omata, N. Murata, Plant Cell Physiol. 24, 1093 (1983).

[33] L. Fiedor, V. Rosenbach-Belkin, A. Scherz, J. Biol. Chem. 267, 22043 (1992).

[34] G. Hartwich, L. Fiedor, I. Simonin, E. Cmiel, W. Schäfer, D. Noy, A. Scherz, H. Scheer, J. Am. Chem. Soc. 120, 3675 (1998).

[35] J.-P. Zhang, H. Nagae, P. Qian, L. Limantara, R. Fujii, Y. Watanabe, Y. Koyama, J. Phys. Chem. B 105, 7312 (2001). 